\title{
碳纤维支撑柔性碳硫复合电极的制备、物性及电池性能研究
}

\author{
李亚东 ${ }^{1}$, 李伟平 ${ }^{1}$ ，王 琴 ${ }^{1}$, 郑道光 ${ }^{1}$, 王建新 ${ }^{2}$ \\ (1. 宁波大学 理学院，宁波 315211 ; 2. 中国科学院 宁波材料技术与工程研究所，宁波 315201)
}

摘 要: 锂硫电池作为极具潜力的下一代二次电池受到广泛关注。然而, 对于含硫正极的研究仍处于实验探索阶段, 商业化的碳纤维延应用于硫正极鲜有报道。本研究制备了锂硫电池用碳纤维支撑柔性碳硫复合电极, 并对其进行了 物性及电池性能的研究。结果发现, 碳纤维段具有多孔隙的三维网络结构, 与具有微孔结构的多孔碳共同构成正极 支撑体, 能够物理固定正极材料, 有助于提高电池的能量密度和锂硫正极的导电性, 界面电阻由原来的 $97.9 \Omega$ 降到 $22.6 \Omega$ 。进一步研究表明, 碳纤维毡做集流体的样品在首圈 $0.05 C$ 倍率下, 具有 $996.7 \mathrm{mAh} / \mathrm{g}$ 的放电比容量, 在 $2 C$ 高倍率下循环 140 圈后仍保持 $666.7 \mathrm{mAh} / \mathrm{g}$ 的放电比容量, 而铝馢样品仅为 772.9 和 $471.6 \mathrm{mAh} / \mathrm{g}$ 。同时, 本研究使 用的 LA132 水系粘结剂、super-P 导电剂价格低廉, 球磨制备工艺可规模化生产、安全环保, 可以为锂硫电池工业 化生产和应用提供参考。

关 键 词: 锂硫电池; 碳纤维支撑; 柔性; 传统铝簿

中图分类号: TQ174 文献标识码: A

\section{Flexible Carbon-fiber Supported Carbon-sulfur Electrode: Preparation, Physical Property and Electrochemical Performance}

\author{
LI Ya-Dong ${ }^{1}$, LI Wei-Ping ${ }^{1}$, WANG Qin ${ }^{1}$, ZHENG Dao-Guang ${ }^{1}$, WANG Jian-Xin ${ }^{2}$ \\ (1. Faculty of Science, Ningbo University, Ningbo 315211, China; 2. Ningbo Institute of Materials Technology and Engineering, \\ Chinese Academy of Sciences, Ningbo 315201, China)
}

\begin{abstract}
Lithium sulfur battery has attracted extensive attention as highly promising next generation of rechargeable battery. However, lithium sulfur battery, especial the sulfur cathode, is still under experimental exploration, and the commercial carbon fiber felt is rarely reported to apply in the sulfur cathode. Herein, the flexible carbon-fibersupported carbon-sulfur electrode was prepared, and its physical properties and electrochemical performance were characterized. The results showed that the interface resistance was reduced from $97.9 \Omega$ to $22.6 \Omega$, due to porous three-dimensional network of the carbon fiber felt and micropore of the porous carbon which benefited energy density of the battery and conductivity of the sulfur cathode. Furthermore, the initial discharge capacity at $0.05 C$ rate was $996.7 \mathrm{mAh} / \mathrm{g}$ and the discharge capacity at $2 C$ rate after 140 cycles was $666.7 \mathrm{mAh} / \mathrm{g}$ when carbon fiber felt serves as current collector, while their counterparts with aluminum foil as the current collector were 772.9 and $471.6 \mathrm{mAh} / \mathrm{g}$, respectively. Furthermore, the environmental-friend and low-cost binder LA132, super-P, and the ball-milling technique are beneficial to mass synthesis, suitable for the industrial production and the application of lithium sulfur battery.
\end{abstract}

Key words: lithium-sulfur battery; carbon-fiber supported; flexible; traditional aluminum foil

收稿日期: 2018-05-21; 收到修改稿日期：2018-09-06

基金项目: 国家自然科学基金(11574168) National Natural Science Foundation of China (11574168)

作者简介: 李亚东(1993-), 男, 硕士研究生. E-mail: liyadong@nimte.ac.cn

通讯作者：王建新，研究员. E-mail: jxwang@nimte.ac.cn 
社会和科技的进步对储能技术提出了更高的要 求, 寻找具有高比能量、长寿命的下一代二次电池 已迫在眉睫。锂硫电池(Lithium-sulfur battery, Li-S battery)因理论比容量高 $(1675 \mathrm{mAh} / \mathrm{g})$ 、能量密度高 $(2600 \mathrm{Wh} / \mathrm{kg})$ 、硫资源储量丰富和环境友好等特点 而被认为是目前最具前景的二次电池 ${ }^{[1-2]}$ 。然而, 单 质硫(Sulfur, $\mathrm{S}_{8}$ ) 和放电产物 $\left(\mathrm{Li}_{2} \mathrm{~S}_{2}\right.$ 和 $\left.\mathrm{Li}_{2} \mathrm{~S}\right)$ 的低电导 率，反应中间产物可溶性多硫化物(LiPS)的穿梭效 应, 负极锂支晶引发的安全问题, 以及充放电过 程中的体积膨胀等, 限制了其商业化发展和实际 应用 ${ }^{[3-5]}$ 。

针对上述锂硫电池的技术问题, 相关团队一直 致力于寻找解决方法, 在正极复合材料的设计和制 备方面已经取得了较大进展。例如, Cui 等 ${ }^{[6]}$ 利用二 氧化钛包覆硫制备的蛋黄式核壳复合材料, 能够有 效缓冲反应过程中的体积膨胀, 抑制穿梭效应。导 电聚合物或双亲性聚合物改性, 包括聚 3,4-乙撑二 氧噻吩 (PEDOT $)^{[7]}$ 、聚吡咯聚乙二醇( ppy-peg) 和聚 乙烯吡咯烷酮(PVP), 能够改善活性物质的导电性, 缓冲体积膨胀 ${ }^{[8-9]}$, 并阻止多硫化物溶解在有机电 解质中。碳质材料如石墨烯 ${ }^{[10-11]}$ 、碳纳米棒 ${ }^{[12]}$ 、碳 纳米管 ${ }^{[13]}$ 、分级多孔碳 ${ }^{[14]}$ 等以及它们的衍生物与单 质硫复合, 利用其多孔和高导电性的特点, 具有物 理固硫和提高硫利用率的作用。其中, 正极的三维 网络结构能够缩短电子和锂离子的传输路径, 为电 化学过程中活性物质硫的转化快速提供电子和锂离 子, 从而提高锂硫电池电化学性能, 受到了广泛关 注 $^{[14-18]}$ 。而到目前为止, 以商业化的碳纤维毡为基 体, 构筑具有三维导电网络结构的含硫正极鲜有 报道。

因此, 本研究以商业化碳纤维廷(Carbon Fiber Felt, CFF) 取代传统铝箔作正极集流体, 并以超级电 容器用多孔碳(Porous Carbon, C) 为硫载体, 充分利 用碳纤维毡和多孔碳的孔隙结构构建三维立体导电 网络, 改善含硫正极的导电性, 进而提升电池性能。 同时, 使用廉价的 super-P 导电剂、LA132 水系粘结 剂以及去离子水为原料, 通过球磨工艺制备锂硫电 池正极浆料, 得到碳纤维毡支撑的柔性碳硫复合电 极, 并研究目标样品的结构、形貌和电化学性能。

\section{1 实验方法}

\section{1 材料合成}

将多孔碳(山东欧铂新材料有限公司)在 $120{ }^{\circ} \mathrm{C}$ 下鼓风干燥, 并与升华硫(上海阿拉丁生化科技股 份有限公司)按照质量比 1:1.5 称量, 于玛瑙研钵中
研磨 $10 \mathrm{~min}$ 以上, 转移至水热反应釜, 在烘箱中以 $5{ }^{\circ} \mathrm{C} / \mathrm{min}$ 的速率升温到 $155{ }^{\circ} \mathrm{C}$, 保温 $24 \mathrm{~h}$ 后冷却 至室温, 得到碳硫粉, 记为 $\mathrm{CS}$ 。将得到的样品在 $\mathrm{N}_{2}$ 氛围下 $250{ }^{\circ} \mathrm{C}$ 处理 $1 \mathrm{~h}$ 得到处理后的碳硫粉, 记 为 $\mathrm{CS}_{\mathrm{v}}{ }^{[19]}$ 。

\section{2 电极制备}

将 $120{ }^{\circ} \mathrm{C}$ 干燥后的 super-P 导电剂(国药集团化 学试剂有限公司)、去离子水混合并磁力搅拌 $30 \mathrm{~min}$ 。 随后与碳硫粉 $\mathrm{CS}_{\mathrm{v}}$ 、直径 $6 \mathrm{~mm}$ 氧化铝磨球置于玛 瑙球磨罐中, 以 $300 \mathrm{r} / \mathrm{min}$ 的转速球磨 $5 \mathrm{~h}$, 添加去 离子水稀释的 LA132 粘结剂(质量比为 $5: 1$ ) (成都 茵地乐电源科技有限公司), 球磨 $1 \mathrm{~h}$, 得到锂硫电 池正极浆料。其中碳硫粉、super-P 导电剂、LA132 的质量比为 $82: 15: 3$, 去离子水与磨球的添加量分 别为浆料固含量的 5 倍和 10 倍。

采用刮刀涂覆工艺，将制备的正极浆料均匀涂 覆在碳纤维毡(日本东丽公司)上, $60{ }^{\circ} \mathrm{C}$ 鼓风干燥 $1 \mathrm{~h}$ 后, 用冲片机冲成直径 $14 \mathrm{~mm}$ 的圆片, $60{ }^{\circ} \mathrm{C}$ 真空干 燥 $12 \mathrm{~h}$, 得到锂硫电池正极片, 标记为 $\mathrm{CFF} / \mathrm{CS}_{\mathrm{v}}$ 。同 时, 制备了以铝箔为集流体的锂硫电池正极片, 标 记为 $\mathrm{Al} / \mathrm{CS}_{\mathrm{v}}$ 。

\section{3 材料表征}

采用 Bruker Axs D8 Advance 型 X 射线衍射仪 ( $\mathrm{CuK} \alpha$ 射线, $\lambda=0.15406 \mathrm{~nm}$ ) 对样品进行组成和物相 分析, 扫描步长 $0.02^{\circ}$, 扫描时间 $0.1 \mathrm{~s} /\left(^{\circ}\right)$; 采用美国 PerkinEimer 公司 Diamond TG/DTA 型热重分析仪在 氩气氛围中对样品进行热重测试, 升温速率 $10^{\circ} \mathrm{C} / \mathrm{min}$; 采用美国 Micromeritics 公司 ASAP 2020 HD88 型全 自动比表面积及微孔孔隙分析仪表征粉体的孔结构 性质(包括比表面积、孔径、孔体积等), 以 $\mathrm{N}_{2}$ 为吸 附介质，测定温度为 $77 \mathrm{~K}$; 采用日立(Hitachi) SU70 型热场发射扫描电子显微镜观察原材料和碳硫复合 正极的微观结构和形貌。

在高纯氩气氛围的手套箱 (水、氧含量低于 $\left.1 \times 10^{-6}\right)$ 中组装 2016 型纽扣电池。以金属锂为负极, 实验制备的极片为正极, 使用商业 Celgard 2400 隔 膜, 电解液 (苏州乾民化学试剂有限公司)配比为 $1 \mathrm{~mol} / \mathrm{L}$ 双三氟甲基磺酸酰亚胺锂(LiTFSI)的乙二醇 二甲醚(DME) 和 1,3-二氧戊环(DOL)(体积比为 1:1) 溶液, 并且添加 $2.0 \mathrm{wt} \% \mathrm{LiNO}_{3}$ 。使用 LAND CT2001A 设备对电池进行充放电性能测试，电压范围为 1.7 $2.8 \mathrm{~V}\left(v s \mathrm{Li} / \mathrm{Li}^{+}\right)$。使用 Bio-Logic VMP3B-20D 电化 学工作站对电池进行循环伏安 (CV)测试(扫描范围 1.6 2.9 V, 速率 $0.1 \mathrm{mV} / \mathrm{s}$ ) 和电化学阻抗(EIS)测试, 频率范围 $10^{-1} \sim 10^{5} \mathrm{~Hz}$ ，振幅为 $5 \mathrm{mV}$ 。 


\section{2 结果与讨论}

\section{1 成分和结构分析}

图 1 为单质硫、多孔碳和碳硫粉的 $\mathrm{X}$ 射线衍射 图谱。观察图 1(a) (c) 发现, 多孔碳与单质硫经过 $155^{\circ} \mathrm{C}$ 熔融处理后, 在 $15.46^{\circ} 、 23.1^{\circ} 、 25.7^{\circ}$ 和 $26.7^{\circ}$ 等处出现明显的单质硫特征峰, 表明实验制备得到 了碳硫复合材料。而观察图 1(d)发现, 经过 $250{ }^{\circ} \mathrm{C}$ 处理 $1 \mathrm{~h}$ 后的碳硫复合材料 $\mathrm{CS}_{\mathrm{v}}$ 未出现明显的单质 硫特征峰, 表明高温处理除去了样品表面附着的大 晶粒硫，使得材料表面没有绝缘性的硫或者仅有很 少量的小晶粒硫残留, 该过程有利于提高碳硫复合 颗粒尤其是颗粒表面的电子电导率 ${ }^{[20-21]}$ 。图 2 为样 品多孔碳、碳硫粉 $\mathrm{CS}$ 和 $\mathrm{CS}_{\mathrm{v}}$ 的热重曲线。观察发 现, 经过 $250{ }^{\circ} \mathrm{C}$ 处理的 $\mathrm{CS}_{\mathrm{v}}$ 失重为 $54.61 \mathrm{wt} \%$, 要低 于未经处理的 $\mathrm{CS}(57.41 \mathrm{wt} \%)$ ，间接表明 $250{ }^{\circ} \mathrm{C}$ 处理 会除去样品表面附着的大晶粒硫 ${ }^{[22]}$ 。

图 3 为原材料多孔碳和实验制得的碳硫复合粉 $\mathrm{CS}_{\mathrm{v}}$ 的氮气吸附-脱附等温曲线，结合表 1 中测得的 数据可知, 原材料多孔碳的比表面积、孔容和平均 孔径分别为 $1734.7 \mathrm{~m}^{2} / \mathrm{g} 、 0.85 \mathrm{~cm}^{3} / \mathrm{g}$ 和 $2.3 \mathrm{~nm}$, 孔结 构以微孔为主。同时, 通过比较发现, 样品 $\mathrm{CS}_{\mathrm{v}}$ 的微 孔比表面积与原材料多孔碳相较显著降低, 原因可 能是材料的微孔中填充了大量的活性物质硫。多孔 碳的大比表面积和孔容为活性物质硫提供了大量的 反应和沉积界面，有利于硫的分散、存储以及对多

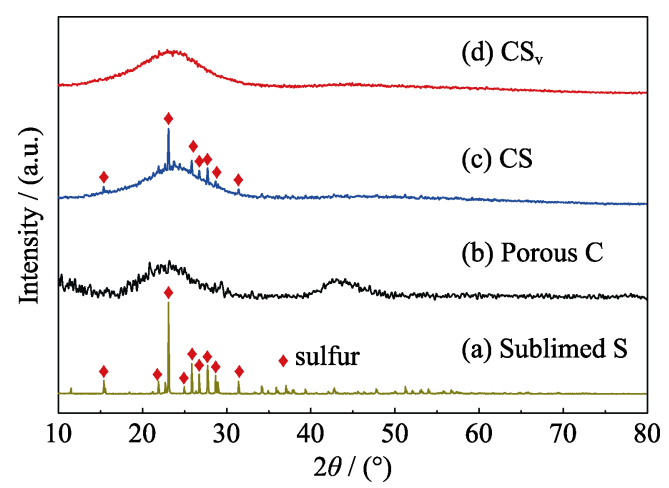

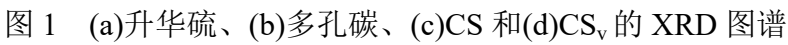

Fig. 1 XRD patterns of (a) sublimed sulfur, (b) porous carbon, (c) $\mathrm{CS}$ and (d) $\mathrm{CS}_{\mathrm{v}}$

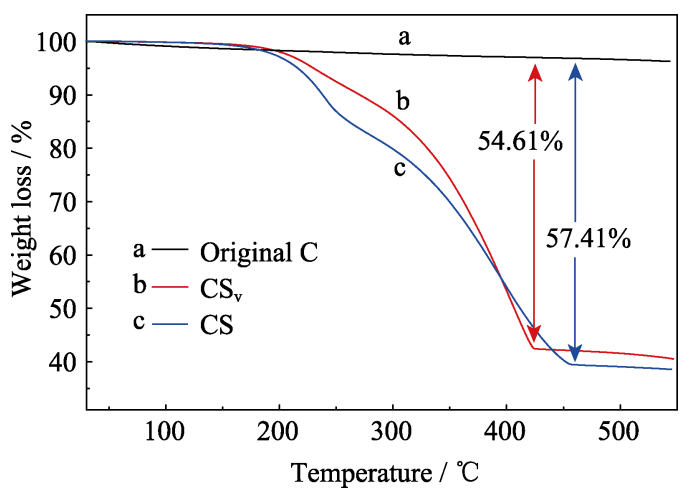

图 2 多孔碳和碳硫粉热重(TG)测试

Fig. 2 Thermal weight (TG) test of porous carbon and carbonsulfur composites

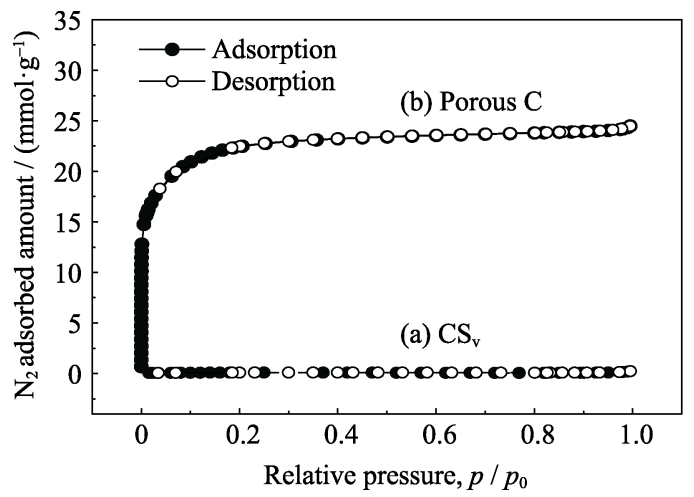

图 3 (a) $\mathrm{CS}_{\mathrm{v}}$ 和(b)多孔碳的氮气吸附-脱附等温曲线

Fig. $3 \mathrm{~N}_{2}$ adsorption-desorption isotherms of (a) $\mathrm{CS}_{\mathrm{v}}$ and (b) porous carbon

硫化锂的物理吸附，有效缓解多硫化物的穿梭效 应。同时也为电池在充放电过程中体积的反复膨胀 收缩提供了空间，使电池结构不易被破坏 ${ }^{[22-24]}$ 。

\section{2 形貌表征}

图 4 为样品的 SEM 照片和 EDS 图谱。由 图 4(a) (c)可知用作正极集流体的碳纤维䏨具有多 孔隙的三维网络结构, 有利于负载正极浆料和储存 电解液，并在活性物质反应过程中起到二次固硫的 作用。观察图 4(d)可知, 实验使用的商业多孔碳平 均粒径小于 $10 \mu \mathrm{m}$ 。观察碳硫粉 $\mathrm{CS}_{\mathrm{v}}$ 的 $\mathrm{EDX}$ 元素分 布图(图 4(h)和(i))可知，硫元素较均匀的分布在碳 硫粉中。同时, 对样品进行了表层元素含量分析 (图 4(j)), 表明硫元素在碳硫粉中含量为 $55 \mathrm{wt} \%$ 左右, 与图 2 的热重测试结果基本一致 ${ }^{[25]}$ 。

表 1 多孔碳的结构特性

Table 1 Texture properties of porous carbon

\begin{tabular}{|c|c|c|c|c|c|c|}
\hline \multirow{2}{*}{ Sample } & \multicolumn{3}{|c|}{ Surface area $/\left(\mathrm{m}^{2} \cdot \mathrm{g}^{-1}\right)$} & \multicolumn{2}{|c|}{ Volume $/\left(\mathrm{cm}^{3} \cdot \mathrm{g}^{-1}\right)$} & \multirow{2}{*}{$\begin{array}{c}\text { Average pore diameter } / \mathrm{nm} \\
\mathrm{BJH} \\
\end{array}$} \\
\hline & $S_{\mathrm{BET}}$ & $S_{\text {micro }}$ & $S_{\text {external }}$ & $V_{\text {total }}$ & $V_{\text {micro }}$ & \\
\hline Porous carbon & 1734.7242 & 1041.1364 & 693.5878 & 0.848649 & 0.472287 & 2.2930 \\
\hline $\mathrm{CS}_{\mathrm{v}}$ & 7.4587 & 1.3661 & 6.0926 & 0.008040 & 0.000438 & 7.1043 \\
\hline
\end{tabular}



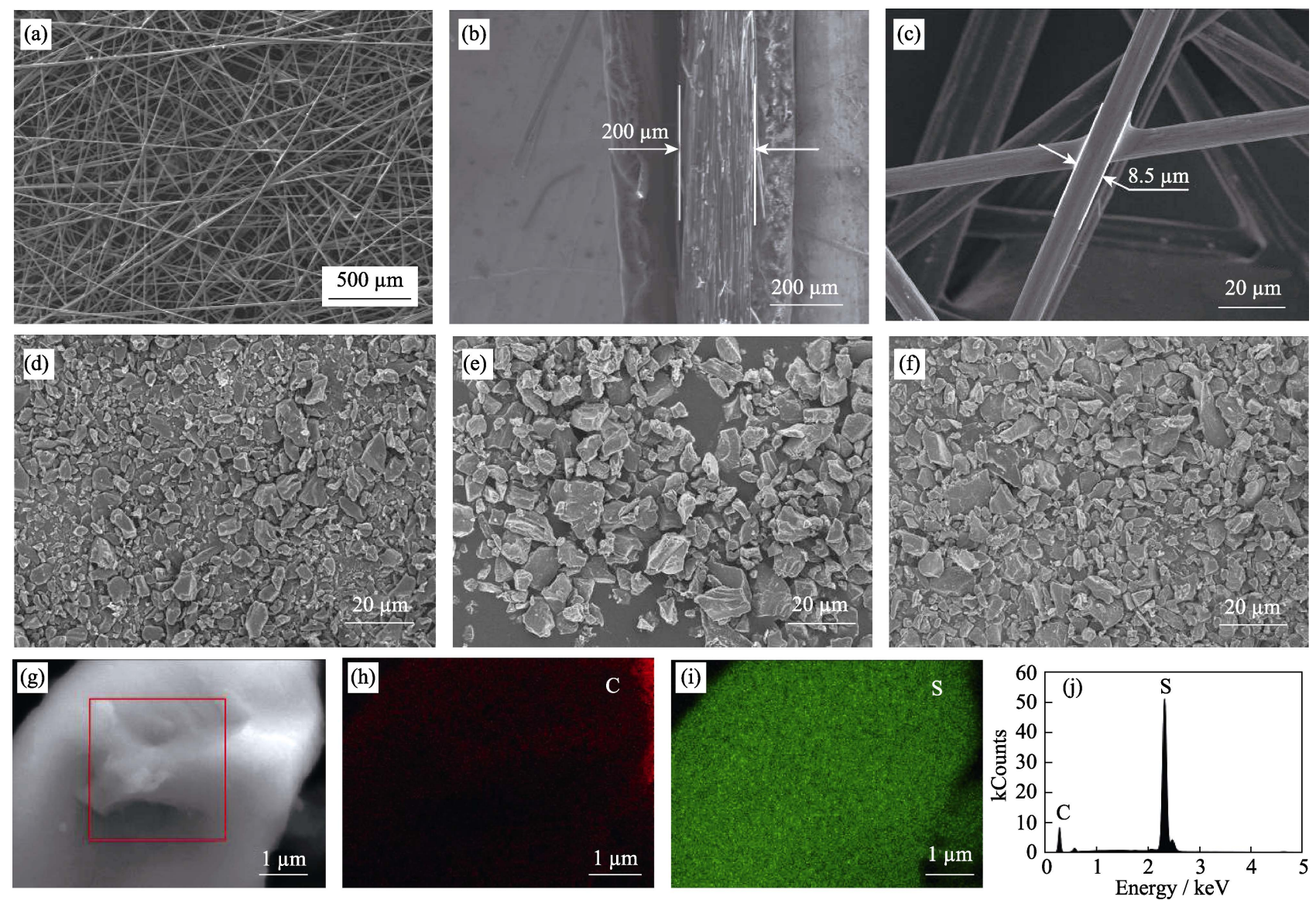

图 4 (a) (c)碳纤维毡、(d)多孔碳、(e) CS、(f) (g) $\mathrm{CS}_{\mathrm{v}}$ 的 SEM 照片; (h) (i) $250{ }^{\circ} \mathrm{C}$ 处理碳硫粉的 EDX 图谱以及 $(\mathrm{j})$ 表面元素含量 $((\mathrm{g})$ 中标识区域)

Fig. 4 SEM images of (a-c) CFF, (d) porous carbon, (e) CS, and (f-g) $\mathrm{CS}_{\mathrm{v}}$, and EDX elemental maps of (h) carbon,

(i) sulfur, and (j) the corresponding mass percentage in the area marked with a rectangle in $(\mathrm{g})$

图 5 是正极片的数码照片以及碳纤维毡和铝簿 分别做集流体的 SEM 照片。如图 5(a) (c) 所示, 实 验制备得到碳纤维支撑的柔性碳硫复合电极, 能够 应用于可穿戴、可弯曲的柔性电子设备。观察图 5(d) 和(e)发现, 正极浆料较均匀的涂覆在碳纤维䏨三维 结构中, 与图 5(f)和 $(\mathrm{g})$ 中铝箔做集流体相比, 碳纤 维毡构成的立体网络结构, 能够物理上固定正极材 料。同时, 碳纤维廷将会充当导电网络的作用, 提高 正极片的导电性和活性物质硫的利用率, 进而提升 锂硫电池充放电性能。并且, 碳纤维䏨做集流体 (厚度 $d=200 \mu \mathrm{m}$, 面密度 $\rho=1.137 \mathrm{mg} / \mathrm{cm}^{2}$ ) 避免了传 统铝箔(厚度 $d=20 \mu \mathrm{m}$, 面密度 $\rho=5.419 \mathrm{mg} / \mathrm{cm}^{2}$ ) 等 引入了密度较大的金属集流体, 有利于提高电池的 能量密度。

\section{3 循环伏安曲线}

如图 6 所示, 碳纤维廷做集流体的电池循环伏 安曲线中, 两个分开的还原峰和两个重叠的氧化峰 与活性物质硫的氧化还原反应相对应。在 2.32 和 $2.0 \mathrm{~V}$ 附近的还原峰分别对应锂硫电池放电过程中 单质硫 $\mathrm{S}_{8}$ 向可溶的长链多硫化锂 $\left(\mathrm{Li}_{2} \mathrm{~S}_{x}, 4 \leqslant x \leqslant 8\right)$ 的
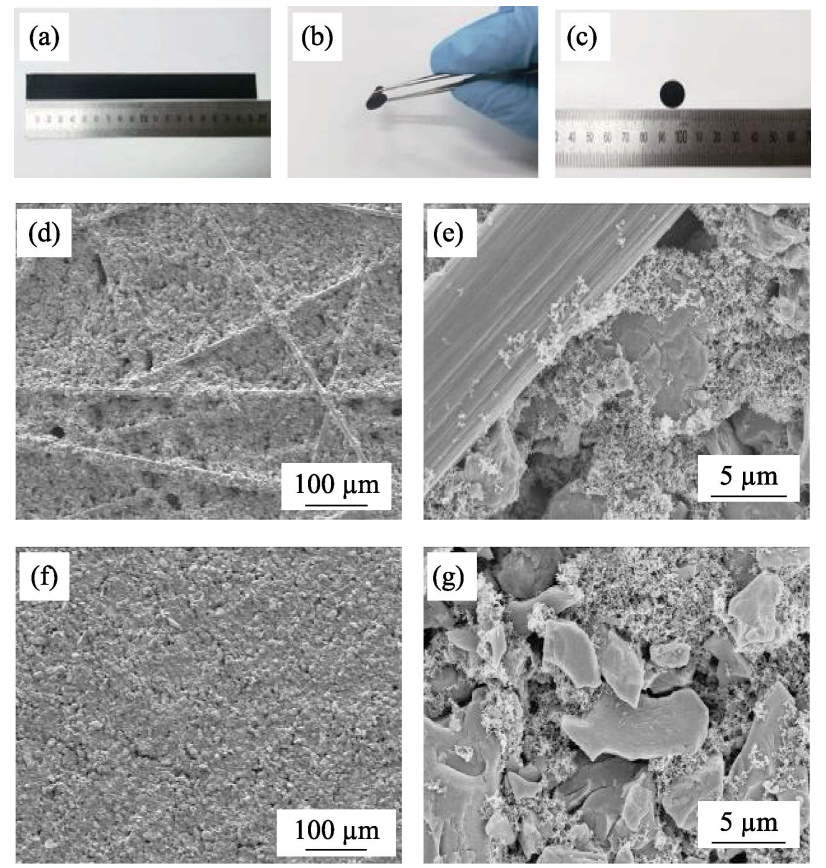

图 5 (a) (e)碳纤维支撑和(f) (g) 铝箔支撑的(a) (c) 正极数 码和(d) (g)SEM 照片,

Fig. 5 (a-c) Digital and (d-g) SEM images of (a-e) CFF/CS and (f-g) $\mathrm{Al} / \mathrm{CS}_{\mathrm{v}}$ cathodes 


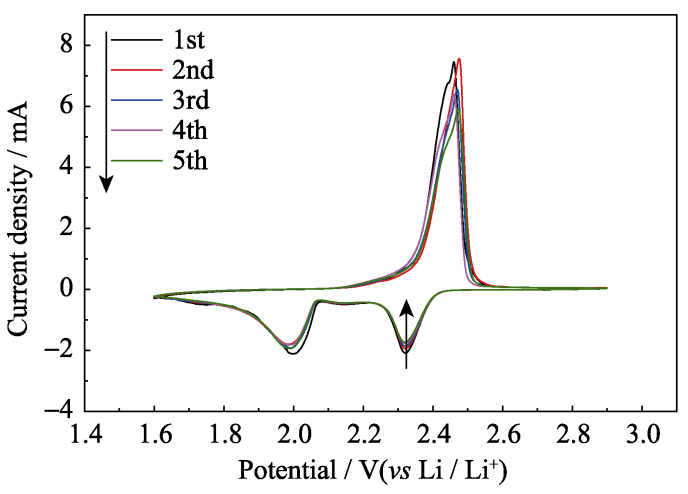

图 $6 \mathrm{CFF} / \mathrm{CS}_{\mathrm{v}}$ 为正极的电池循环伏安曲线

Fig. 6 Typical cyclic voltammograms of devices with $\mathrm{CFF} / \mathrm{CS}_{\mathrm{v}}$ as cathode

转变和长链多硫化锂向不溶的短链硫化物 $\left(\mathrm{Li}_{2} \mathrm{~S}_{2} /\right.$ $\left.\mathrm{Li}_{2} \mathrm{~S}\right)$ 的转变 ${ }^{[26-27]}$ 。在 $2.45 \mathrm{~V}$ 附近的氧化峰对应电池 充电过程中 $\mathrm{Li}_{2} \mathrm{~S}_{2} / \mathrm{Li}_{2} \mathrm{~S}$ 向单质硫或者长链多硫化锂 转变的反应 ${ }^{[28]}$ 。

\section{4 电化学交流阻抗测试}

图 7 显示了碳纤维䏨和铝箔分别做正极集流体, 并在组装后静置 $12 \mathrm{~h}$ 的电池电化学阻抗图谱。等效 电路中的 $R_{\mathrm{e}}$ 代表电解质电阻, $R_{1}$ 代表界面阻抗并对 应阻抗图谱中高频区的半圆大小, $W_{\mathrm{o}}$ 代表 Warburg 阻抗, CPE 代表恒相位元 ${ }^{[19,29-30]}$ 。观察发现, 铝䈃样 品电池的界面阻抗为 $97.9 \Omega$, 而碳纤维毡样品电池 仅为 $22.6 \Omega$, 电池阻抗显著降低。这是由于从传统 铝䈃做集流体的面导电结构转变为碳纤维毡的三维 立体导电结构, 形成了三维互连的电子通路和相互 连通的离子扩散通道，从而提高了正极的导电性。

\section{5 电池循环性能测试}

图 8(a)对比了碳纤维毡和铝箔分别做锂硫正极 集流体时不同倍率下的电池循环性能。观察发现， 与铝箔相比，在相同放电倍率和更高面积载硫量条

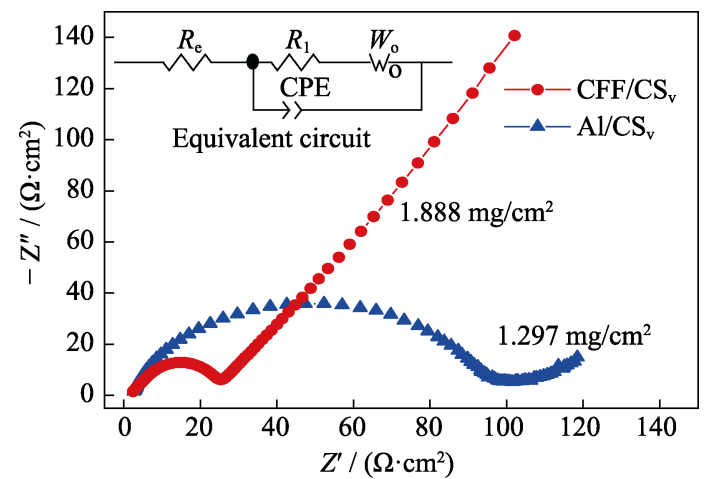

图 7 碳纤维毡和铝䇴分别做集流体的电池阻抗, 插图为等 效电路图

Fig. 7 Electrochemical impedance plots of the batteries with $\mathrm{Al} / \mathrm{CS}_{\mathrm{v}}$ and $\mathrm{CFF} / \mathrm{CS}_{\mathrm{v}}$ as the current collectors with inset showing equivalent circuit
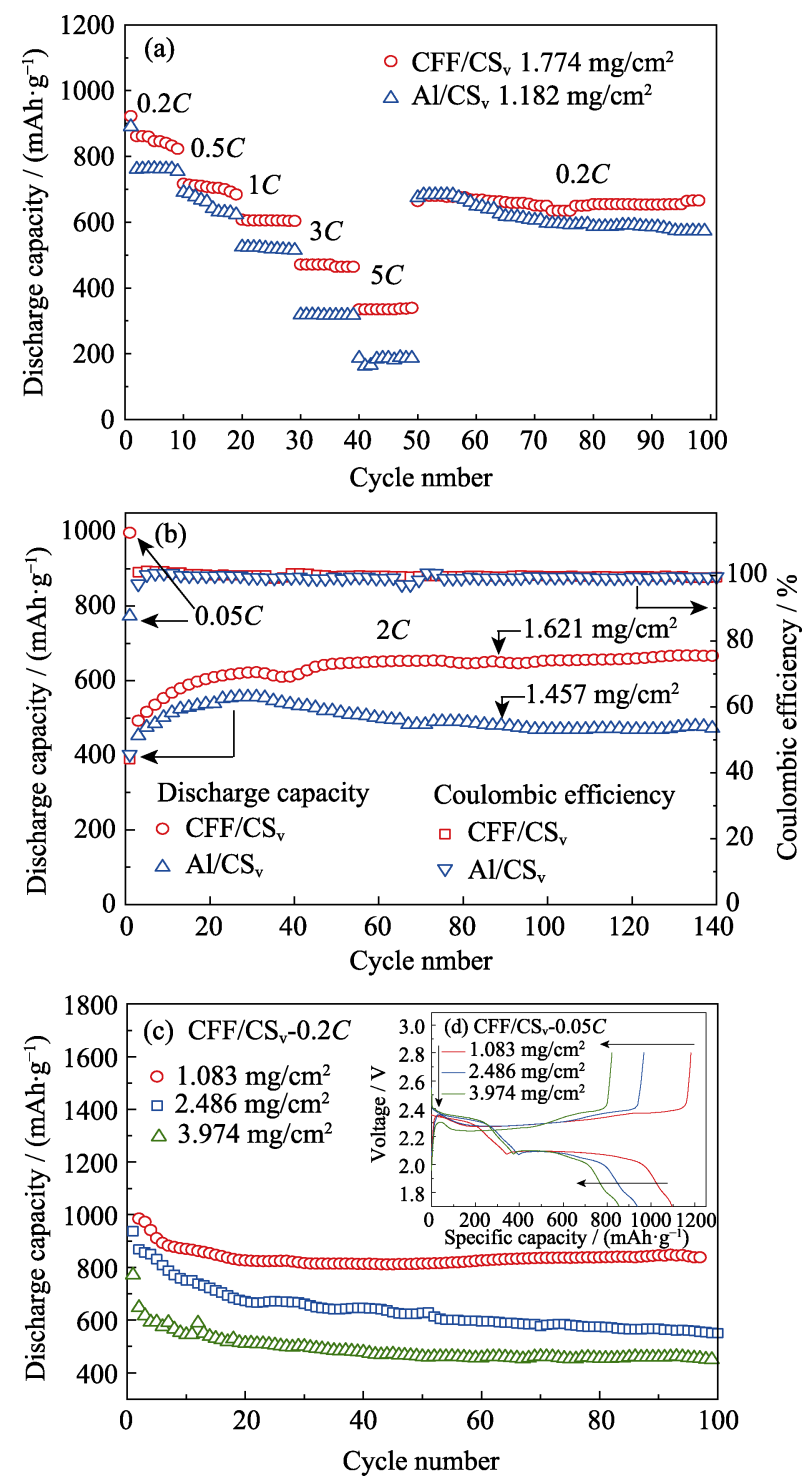

图 8 碳纤维毡和铝箔分别做集流体的电池在(a) $0.2 C \sim 5 C$ 和 (b) $2 C$ 下的循环性能曲线; 不同硫负载量碳纤维毡做集流体 的电池在 $(\mathrm{c}) 0.2 \mathrm{C}$ 下的循环性能曲线和(d) 首圈 $0.05 C$ 下的充 放电曲线

Fig. 8 Cycle performance of $\mathrm{CFF} / \mathrm{CS}_{\mathrm{v}}$ and $\mathrm{Al} / \mathrm{CS}_{\mathrm{v}}$ batteries at (a) $0.2 C-5 C$ rates and (b) $2 C$ rate, (c) Cycle performance of $\mathrm{CFF} / \mathrm{CS}_{\mathrm{v}}$ batteries with different $\mathrm{S}$ load at $0.2 \mathrm{C}$ rate, and (d) charge-discharge performance of the $\mathrm{CFF} / \mathrm{CS}_{\mathrm{v}}$ batteries with different $\mathrm{S}$ load at $0.05 C$ rate

件下, 碳纤维毡样品表现出更优异的循环性能。图 8(b) 为碳纤维廷和铝䈃样品在 $2 C(1 C=1675 \mathrm{~mA} / \mathrm{g})$ 倍率 下的循环性能，其中前 20 圈容量增加可能是高倍率 下电池活化过程缓慢，未反应的活性物质硫随着反 应的进行逐渐参与反应所致。观察图 8(b)发现，正 极面积载硫量为 $1.621 \mathrm{mg} / \mathrm{cm}^{2}$ 时, 碳纤维毡样品首 圈 $0.05 C$ 倍率下, 具有 $996.7 \mathrm{mAh} / \mathrm{g}$ 的放电比容量, 并在 $2 C$ 倍率下循环 140 圈后仍具有 $666.7 \mathrm{mAh} / \mathrm{g}$ 的放电比容量，而相同条件下铝箔样品仅为 772.9 和 $471.6 \mathrm{mAh} / \mathrm{g}$, 电池循环性能显著提高。 
碳纤维支撑碳硫复合电极相比于传统铝箔能够 明显提高电池充放电性能，原因可能是碳纤维毡的 三维立体导电结构有利于提高含硫正极导电性和活 性物质利用率。同时, 如图 8(c)和插图(d)所示, 实 验研究了碳纤维毡样品不同硫负载量时, 首圈 $0.05 C$ 倍率下的充放电曲线和 $0.2 C$ 倍率下的循环性 能。结果发现, 随着硫负载量增加, 电池首圈和循环 10 圈后的放电比容量逐渐降低, 但在 $3.974 \mathrm{mg} / \mathrm{cm}^{2}$ 高负载量和 $0.2 C$ 倍率下，电池循环 100 圈后仍保持 $449.2 \mathrm{mAh} / \mathrm{g}$ 的放电比容量。

\section{3 结论}

实验制备了具有三维网络结构的碳纤维支撑柔 性碳硫复合电极。碳纤维毡的多孔隙结构能够物理 上固定正极材料。三维立体导电结构形成的互连电 子通路可以提高正极导电性, 进而提升锂硫电池充 放电性能。结果表明, 电池阻抗显著降低, 由铝箔样 品的 $97.9 \Omega$ 降为碳纤维毡样品的 $22.6 \Omega$; 电池循环 性能显著提高, 在正极面积载硫量为 $1.621 \mathrm{mg} / \mathrm{cm}^{2}$ 时, $2 C$ 倍率下循环 140 圈后仍具有 $666.7 \mathrm{mAh} / \mathrm{g}$ 的 放电比容量, 而铝箔样品仅为 $471.6 \mathrm{mAh} / \mathrm{g}$ 。

\section{参考文献:}

[1] YANG Y, ZHENG G Y, CUI Y. Nanostructured sulfur cathodes. Chemical Society Reviews, 2013, 42(7): 3018-3032.

[2] ROSENMAN A, MARKEVICH E, SALITRA G, et al. Review on Li-sulfur battery systems: an integral perspective. Advanced Energy Materials, 2015, 5(11): 1500212-1-21.

[3] ZHANG R, CHENG X B, ZHAO C Z, et al. Conductive nanostructured scaffolds render low local current density to inhibit lithium dendrite growth. Advanced Material, 2016, 28(11): 2155-2162.

[4] CHENG X B, ZHANG R, ZHAO C Z, et al. A review of solid electrolyte interphases on lithium metal anode. Advanced Material, 2016, 3(3): 1500213-1-20.

[5] MARMORSTEIN D, YU T H, STRIEBEL K A, et al. Electrochemical performance of lithium/sulfur cells with three different polymer electrolytes. Journal of Power Sources, 2000, 89(2): 219-226.

[6] LI W Y, ZHENG G Y, CUI Y, et al. Sulphur-TiO 2 yolk-shell nanoarchitecture with internal void space for long-cycle lithiumsulphur batteries. Nature Communications, 2013, 4(4): 1331-1-6.

[7] CHEN H W, DONG W L, WANG C, et al. Ultrafine sulfur nanoparticles in conducting polymer shell as cathode materials for high performance lithium/sulfur batteries. Scientific Reports, 2013, 3(5): 1910-1-6.

[8] WU F, CHENG J Z, CHENG R J, et al. Polyethylene-glycol-doped polypyrrole increases the rate performance of the cathode in lithiumsulfur batteries. ChemSusChem, 2013, 6(8): 1438-1444.

[9] DIRLAM P T, CHAR K, PYUN J, et al. The use of polymers in Li-S batteries: a review. Journal of Polymer Science A: Polymer Chemistry, 2017, 55: 1635-1668.

[10] PENG H J, HUANG J Q, WEU F, et al. Nanoarchitectured graphene/ CNT@porous carbon with extraordinary electrical conductivity and interconnected micro/mesopores for lithium-sulfur batteries. Advanced Functional Materials, 2014, 24(19): 2772-2781.

[11] ZHANG Z, KONG L L, LIU S, et al. A high-efficiency sulfur/ carbon composite based on 3D graphene nanosheet@carbon nanotube matrix as cathode for lithium-sulfur battery. Advanced Energy Materials, 2017, 7(11): 1602543-1-12.

[12] ZENG L C, YAO Y, YU Y, et al. A flexible $\mathrm{S}_{1_{-}} \mathrm{Se}_{x} @$ porous carbon nanofibers $(x \leqslant 0.1)$ thin film with high performance for Li-S batteries and room-temperature $\mathrm{Na}-\mathrm{S}$ batteries. Energy Storage Materials, 2016, 5: 50-57.

[13] HANG S C, SONG M S, LEE J Y, et al. Effect of multiwalled carbon nanotubes on electrochemical properties of lithium/sulfur rechargeable batteries. Journal of Electrochemical Society, 2003, 150(7): A889-A893.

[14] CHANG Z, DING B, DOU H, et al. Hierarchically porous multilayered carbon barriers for high-performance $\mathrm{Li}-\mathrm{S}$ batteries. Chemistry, 2018, 24(15): 3768-3775.

[15] LI G R, LEI W, CHEN Z W, et al. 3D porous carbon sheets with multidirectional ion pathways for fast and durable lithium-sulfur batteries. Advanced Energy Materials, 2018, 8(8): 1702381-1-10.

[16] CHUNG S H, MANTHIRAM A. Low-cost, porous carbon current collector with high sulfur loading for lithium-sulfur batteries. Electrochemistry Communications, 2014, 38: 91-95.

[17] HU M M, HU T, LI Z J, et al. Surface functional groups and interlayer water determine the electrochemical capacitance of $\mathrm{Ti}_{3} \mathrm{C}_{2} \mathrm{~T}_{x}$ MXene. ACS Nano, 2018, 12(4): 3578-3586.

[18] YANG W, SONG A L, SUN G, et al. 3D interconnected porous carbon nanosheets/carbon nanotubes as a polysulfide reservoir for high performance lithium-sulfur batteries. Nanoscale, 2018, 10(2): 816-824.

[19] ZHAO Z X, QING D, WANG S, et al. Fabrication of high conductive $\mathrm{S} / \mathrm{C}$ cathode by sulfur infiltration into hierarchical porous carbon/ carbon fiber weave-structured materials via vapor-melting method. Electrochimica Acta, 2014, 127: 123-131.

[20] LI X, WANG L J, XIA, LIU Z, et al. Catalytic oxidation of toluene over copper and manganese based catalysts: effect of water vapor. Catalysis Communications, 2011, 14(1): 15-19.

[21] CUI X L, SHAN Z Q, CUI L, et al. Enhanced electrochemical performance of sulfur/carbon nanocomposite material prepared via chemical deposition with a vacuum soaking step. Electrochimica Acta, 2013, 105(26): 23-30.

[22] SCHUSTER J, YIM T, NAZAR L F, et al. Spherical ordered mesoporous carbon nanoparticles with high porosity for lithiumsulfur batteries. Angewandte Chemie International Edition, 2012, 51(15): 3591-3595.

[23] NAZAR L F, JI X L, LEE K T. A highly ordered nanostructured carbon-sulphur cathode for lithium-sulphur batteries. Nature $\mathrm{Ma}$ terials, 2009, 8(6): 500-506.

[24] OSCHATZ M, THIEME S, BORCHARDT L, et al. A new route for the preparation of mesoporous carbon materials with high performance in lithium-sulphur battery cathodes. Chemical Communications, 2013, 49(52): 5832-5834.

[25] YI L L, WANG X Y, WANG G, et al. Improved electrochemical performance of spherical $\mathrm{Li}_{2} \mathrm{FeSiO}_{4} / \mathrm{C}$ cathode materials via $\mathrm{Mn}$ doping for lithium-ion batteries. Electrochimica Acta, 2016, 222: 1354-1364.

[26] LI G C, HU J J, LI G R, et al. Sulfur/activated-conductive carbon black composites as cathode materials for lithium/sulfur battery. Journal of Power Sources, 2013, 240(31): 598-605.

[27] YAMIN H, GORENSHTEIN A, PENCINER J, et al. Lithium sulfur battery: oxidation/reduction mechanisms of polysulfides in THF solutions. Journal of Electrochemical Society, 1988, 19(33): $1045-1048$.

[28] RAUH R D, ABRAHAM K M, PEARSON G F, et al. A lithium/ dissolved sulfur battery with an organic electrolyte. Journal of Electrochemical Society, 1979, 126(4): 523-527.

[29] KOLOSNITYN V S, KUZMINA E V, KARASEVA S E, et al. A study of the electrochemIcal processes in lithium-sulphur cells by impedance spectroscopy. Journal of Power Sources, 2011, 196(3): $1478-1482$.

[30] XIE J, YANG J, ZHOU X, et al. Preparation of three-dimensional hybrid nanostructure-encapsulated sulfur cathode for high-rate lithium sulfur batteries. Journal of Power Sources, 2014, 253(5): $55-63$. 